\title{
An Effective Approach for Uncertain Aerodynamic Analysis of Airfoils via the Polynomial Chaos Expansion
}

\author{
Xufang Zhang $\mathbb{D}^{1}$ and Jiafei Sun ${ }^{2}$ \\ ${ }^{1}$ School of Mechanical Engineering \& Automation, Northeastern University, Shenyang, Liaoning 110819, China \\ ${ }^{2}$ BYD Auto Industry Co. Ltd., Shenzhen, Guangdong 518118, China \\ Correspondence should be addressed to Xufang Zhang; zhangxf@mail.neu.edu.cn
}

Received 19 September 2019; Revised 11 November 2019; Accepted 17 December 2019; Published 26 February 2020

Academic Editor: Luca Chiapponi

Copyright ( $\odot 2020$ Xufang Zhang and Jiafei Sun. This is an open access article distributed under the Creative Commons Attribution License, which permits unrestricted use, distribution, and reproduction in any medium, provided the original work is properly cited.

This paper presents an effective approach for uncertain aerodynamic analysis of airfoils via the polynomial chaos expansion (PCE). To achieve this, the multivariate polynomial is first setup to represent random factors within the aerodynamic model, whereas the expansion coefficient is expressed as the multivariate stochastic integral of the input random vector. In this regard, the statistical regression in conjunction with a small number of representative samples is employed to determine the expansion coefficient. Then, a combination of the PCE surrogate model with brutal-force Monte Carlo simulation allows to determine numerical results for the uncertain aerodynamic analysis. Potential applications of this approach are first illustrated by the uncertainty analysis of the Helmholtz equation with spatially varied wave-number random field, and its effectiveness is further examined by the uncertain aerodynamic analysis of the NACA 63-215 airfoil. Results for the small regression error and a close agreement between simulated and benchmark results have confirmed numerical accuracy and efficiency of this approach. It, therefore, has a potential to deal with computationally demanding aerodynamical models for the uncertainty analysis.

\section{Introduction}

With the fast development of computer and simulation techniques, numerical methods for the aerodynamic analysis of airfoils have been received considerable attentions during past decades $[1,2]$. Various simulation codes based on the finite difference, the finite element, and the finite volume were extensively investigated $[3,4]$. In this regard, parameters of the aerodynamic model, i.e., the geometry dimensions and the boundary conditions are usually assumed as deterministic [5-7].

However, the uncertainty is universally existed in aerodynamic models of an airfoil [8-10]. Statistical characteristics of the average wind velocity and the turbulence intensity are necessarily described by using the probability distribution [11]. In this regard, Ernst et al. [12] considered geometric uncertainties of rotor blades that arise from manufacturing tolerances and operational wears, and the input uncertainty indeed leads to a significant scatter of the lift and drag coefficients. Zhu and Qiu [13] investigated the possibility of utilizing perturbation approaches for uncertain aerodynamic loads modelling, and their combined effects on the response interval prediction of lift coefficients.

Rather than utilizing the first-order or the Neumann series to approximate the aerodynamic response function, the Monte Carlo simulation can deal with the uncertain aerodynamic analysis in a much more straightforward manner [14]. With a large number of samples to represent the input uncertainty, the question of dealing with the uncertain aerodynamic analysis is degenerated as a series of deterministic model evaluations [15]. This routine can be applied for the moment analysis, the prediction of response distribution, and the global sensitivity analysis $[16,17]$. Therefore, combined with the parallel computing technology, results determined by the Monte Carlo simulation method is assumed to provide benchmarks for uncertainty aerodynamic analysis of airfoils in this paper.

However, the convergence rate of the Monte Carlo simulation (MCS) method is fairly slow, i.e., $1 / \sqrt{N_{M C S}}$ for a total of $N_{\text {MCS }}$ samples used in this simulation [18]. This 
implies that a large number of random samples are necessary for an accurate estimation result. If each round of the deterministic aerodynamic analysis is relatively time consuming, e.g., about three minutes, the overall uncertain aerodynamic analysis with $10^{4}$ samples would be about 20.83 days. This motivates an efficient approximation method for the uncertainty aerodynamic analysis in this paper.

Besides the brutal-force random sampling method, several improved versions of the MCS based on low-discrepancy points [19], the Quasi Monte Carlo method [20], and the active-learning approach $[21,22]$ were alternatively developed. It can improve numerical efficiency of the random simulation method to some extents. However, this paper specially focuses on the polynomial chaos expansion method, which is able to provide a global approximation result of the mechanistic model for the uncertain aerodynamic analysis of airfoils.

The ideal of the polynomial chaos expansion was initially introduced by Wiener [23] to investigate the Gaussian turbulence models. Li and Ghanem [24] further investigated the utility of the chaos expansion approach for the stochastic finite element analysis of various solid mechanics models [25]. Combined with the Askey scheme, Xiu and Karniadakis [26] extended this expansion approach to deal with continuous random variables. To achieve this, the basis functions for input random variables are first constructed by using the chaotic rule of marginal orthogonal polynomials [27]. This allows to represent a multivariate model with the generalized Fourier series expansion [28]. Once the PCE model for an airfoil model is determined, the Gaussian quadrature approach can be used to deal with multivariate integrals on the expansion coefficient [29-31]. This results in the Galerkin projection [32], the stochastic collocation [33-35], and the statistical regression methods [36] in the literature.

In general, numerical algorithms for this PCE model are divided as the intrusive and nonintrusive approaches. The first category requires to continuously revise the deterministic aerodynamic model, which is time consuming and difficult for complex simulation models [37]. In the contrast, the nonintrusive approach treats the deterministic aerodynamic analysis as a black-box function and represents random variables as a limited number of deterministic values. After substituting for representative values of a random variable, statistical characteristics of the uncertain aerodynamic response can be obtained based on a simple postprocessing procedure in terms of some deterministic analyses.

Since a large number of aerodynamic analyses are embedded in the brutal-force Monte Carlo simulation, this generally results in computationally demanding cost. The PCE approach was combined with the Gaussian quadrature scheme and the dimensional reduction method [38]. However, the standard Gaussian method might result in the problem of the curse of dimensionality [39], which motives the nonintrusive and the statistical regression method to determine expansion coefficients in this paper.
With low-discrepancy samples generated based on the Sobol' or the Latin Hypercube scheme, the estimation of PCE coefficients can be realized by the generalized statistical regression procedure as shown in numerical examples. Even though sparse regression algorithms based on the orthogonal matching pursuit [40], the backward-and-forward selection, and the least-angle regression [41] can be alternatively realized, the paper specially focuses on the utility of the full PCE model and the standard regression approach due to its robustness.

To summarize, an objective of this paper is to present an effective approach for uncertainty aerodynamic analysis of airfoil models via the polynomial chaos expansion. A statistical regression method in conjunction with the lowdiscrepancy samples is employed to determine the PCE coefficient. To demonstrate potential applications of this method, examples in the literature are presented to examine its numerical performance.

The rest of the manuscript is organized as follows. Section 2 briefly summarizes the method of the polynomial chaos expansion for the uncertain aerodynamic analysis of an airfoil, and a statistical regression approach is presented in Section 2.3 to determine the expansion coefficient. Section 3 illustrates an application of this method for uncertain analysis of the Helmholtz equation with a spatially varying wave-number model. Results for uncertain aerodynamic analysis of the NACA 63-215 airfoil are discussed in Section 4 , and conclusions are summarized in Section 5.

\section{Uncertainty Analysis via the Polynomial Expansion Method}

The aerodynamic response of an airfoil (e.g., the pressure and the velocity field and the lift or the drag coefficient) would become stochastic, if input random variables are considered in the model function $\eta(\mathbf{X} ; u, v, w)$. Herein, $u, v$, and $w$ represent three physical dimensions of the aerodynamic model $\eta(\cdot)$, whereas the random vector $\mathbf{X}=$ $\left[X_{1}, \cdots, X_{d}\right]^{\mathrm{T}}$ consists of all uncertain factors. Examples of the random variable include the average wind speed, the air density, and the viscosity parameters, as shown in numerical examples. To account for this input uncertainty, a regression-based polynomial chaos expansion method is presented as follows.

2.1. A Review of the Polynomial Chaos Expansion. To develop the polynomial chaos expansion model for the aerodynamic analysis, we first define an index vector $\boldsymbol{\alpha}=\left[\alpha_{1}, \cdots, \alpha_{d}\right]^{\mathrm{T}}$ with each integer $\alpha_{i} \in[0, p]$. Then, a set of chaos polynomials defined by utilizing the highest order-parameter $p$ and $d$-dimensional random vector $\mathbf{X}$ are expressed as

$$
\left\{\phi_{i}(\mathbf{X}), i=0, \ldots, n-1\right\}:=\bigcup_{|\alpha| \leq p} \prod_{k=1}^{d} \varphi_{\alpha_{k}}\left(X_{k}\right) .
$$

Herein, $X_{k}$ is $k$ th input random variable, whereas $\varphi_{\alpha_{k}}\left(X_{k}\right)$ denotes $\alpha_{k}$ th-order orthogonal polynomials that is used to represent the random variable $X_{k}$. 
Specially, the order of a $d$-variate polynomial is defined as $p=\sum_{k=1}^{d} \alpha_{k}$, and the number of elements within the polynomial set $\left\{\phi_{i}(\mathbf{X})\right\}_{i=0}^{n-1}$ is [24]

$$
n=\left(\begin{array}{c}
d+p \\
p
\end{array}\right)=\frac{(d+p) !}{d ! p !}
$$

Besides, arbitrary two elements within the polynomial set $\left\{\phi_{i}(\mathbf{X}), i=0, \ldots, N-1\right\}$ are orthogonally defined. This is $\left\langle\phi_{i}(\mathbf{x}), \phi_{j}(\mathbf{x})\right\rangle=\delta_{i j}$ (as $\left.i, j=\cdots, n\right)$, where $\delta_{i j}$ denotes the Kronecker delta symbol. Therefore, the chaotic polynomial set $\left\{\phi_{i}(\mathbf{X}), i=0,1, \ldots, n-1\right\}$ constitute a complete set to represent a $d$-dimensional real-valued space when $n \longrightarrow+\infty$, and the structural model response can be spectrally represented as

$$
\eta(\mathbf{X} ; u, v, w)=\sum_{i=0}^{\infty} a_{i}(u, v, w) \phi_{i}(\mathbf{X})
$$

Herein, $a_{i}(u, v, w)$ denotes deterministic expansion coefficients:

$$
a_{i}(u, v, w)=\int_{\mathbf{X}} \phi_{i}(\mathbf{x}) \eta(\mathbf{X} ; u, v, w) f_{\mathbf{X}}(\mathbf{x}) \mathrm{d} \mathbf{x}, \quad i=0, \ldots, n-1
$$

Herein, $f_{\mathbf{X}}(\mathbf{x})$ denotes the joint probability density function the input random vector $\mathbf{X}$.

Since the PCE model contains infinite terms in the definition, the approximation for an aerodynamic response can be numerically realized by truncating the expression starting an $n$th term:

$$
\widehat{\eta}(\mathbf{X} ; u, v, w)=\sum_{i=0}^{n-1} a_{i}(u, v, w) \phi_{i}(\mathbf{X})
$$

which is used to approximate the true mechanistic model in numerical simulations.

The normalization constant of Jacobi polynomials: $C_{\text {Jacobi }}=\left(2^{a+b+1} / 2 i+a+b+1\right) \quad(\Gamma(i+a+1) \Gamma(i+b+1) /$ $\Gamma(i+a+b+1) i !)$.

Specially, the orthogonal chaos polynomial depends largely on the probability distribution of input random variables. Table 1 summarizes the relation between the probability distribution of random variables and the orthogonal polynomial used to develop the PCE model. Once the spacial-dependent coefficients in equation (4) are numerically determined, uncertain response of the aerodynamic model $\eta(\mathbf{X} ; u, v, w)$ can be theoretically represented by the truncated PCE model. Therefore, numerical approaches for the calculation of the PCE coefficient are presented as follows.

2.2. The Calculation of PCE Coefficients. The key issue to realize the PCE method for the uncertainty quantification is to determine expansion coefficients $a_{k}(u, v, w)$ $(k=0,1, \cdots, n-1)$ in equation (5), which are generally modelled by using the following optimization problem: $\begin{cases}\text { Find: } & \left\{\widehat{a}_{0}(u, v, w), \widehat{a}_{1}(u, v, w), \cdots, \widehat{a}_{n-1}(u, v, w)\right\} \\ \text { Minimize: } & \left\|\eta\left(\mathbf{x}^{(k)}\right)-\sum_{i=0}^{N-1} \widehat{a}_{i}(u, v, w) \phi_{i}\left(\mathbf{x}^{(k)}\right)\right\|_{q}\end{cases}$

Herein, $\mathbf{x}^{(k)}$ represents the $k$ th realization of the input random vector $\mathbf{X}$, whereas $\|\cdot\|_{q}$ denotes a $q$-norm operator with respect to totally $n_{0}$ residual terms of $\eta(\cdot)$ as referring to the PCE approximation result $\hat{\eta}(\cdot)$.

If the parameter $q=2$ and $n_{0}=n$, the probabilistic optimization problem directly leads to an $n$-order algebraic system in term of the PCE coefficients:

$$
\begin{array}{r}
\left\langle\left[\eta(\mathbf{X} ; u, v, w)-\sum_{k=0}^{n-1} a_{k}(u, v, w)\left\langle\phi_{k}(\mathbf{X})\right], \phi_{i}(\mathbf{X})\right\rangle=0\right. \\
i=0, \ldots, n-1
\end{array}
$$

which can be re-expressed as

$$
\begin{array}{r}
\left\langle\eta(\mathbf{X} ; u, v, w), \phi_{i}(\mathbf{X})\right\rangle=\sum_{k=0}^{n-1} a_{k}(u, v, w)\left\langle\phi_{k}(\mathbf{X}), \phi_{i}(\mathbf{X})\right\rangle, \\
i=0, \ldots, n-1 .
\end{array}
$$

Considering that $\left\langle\phi_{k}(\mathbf{X}), \phi_{i}(\mathbf{X})\right\rangle=\delta_{i k} \quad$ as $i, k=0, \ldots, n-1$, this finally derives totally $n d$-dimensional integrals as expressed in equation (4).

The brutal-force MCS method and its optimized versions have been used in the literature to deal with the high-dimensional integration. Results for the MCS based estimation of the expansion coefficient are

$$
\begin{array}{r}
\widehat{a}_{i}(u, v, w) \approx \frac{1}{N_{\mathrm{MCS}}} \sum_{j=1}^{N_{\mathrm{MCS}}} \eta\left(\mathbf{x}^{(j)} ; u, v, w\right) \phi_{i}\left(\mathbf{x}^{(j)}\right) \\
i=0, \ldots, n-1
\end{array}
$$

where the symbol $\mathbf{x}^{(i)}$ represents the $i$ th realization of the input random vector $\mathbf{X}$ and $N_{\text {MCs }}$ represents the total number of samples used in the Monte Carlo simulation.

Note that numerical realization of such large number of high-dimensional integrals is a computationally intensive task, given that the deterministic aerodynamic response $\eta(\mathbf{X} ; u, v, w)$ is possibly spatially modelled based on a finite element scheme. In this regard, the brutal-force MCS method is replaced with the statistical regression method as follows.

2.3. The Statistical Regression Method. Denote that $\zeta=\left\{\mathbf{x}^{(1)}, \cdots, \mathbf{x}^{(m)}\right\}$ consists of $m$ realizations of the input random vector $\mathbf{X}$ generated based on a quasi-simulation method in conjunction with a low-discrepancy sequence (such as the Sobol', Halton, or Hammersley). Corresponding numerical realizations of the chaos polynomial set $\left\{\phi_{i}(\mathbf{x})\right\}_{i=0}^{n-1}$ would be 
TABLE 1: Distribution of random variables and orthogonal polynomials [26].

\begin{tabular}{lcccc}
\hline Polynomial & Weight function & Domain & Orthogonality & Distribution \\
\hline Hermit & $\exp \left(-x^{2} / 2\right)$ & $(-\infty,+\infty)$ & $\left\langle\psi_{i}, \psi\right\rangle_{j}=\sqrt{2 \pi i !} \delta_{i j}$ & Normal \\
Legendre & 1 & {$[-1,1]$} & $\left\langle\psi_{i}, \psi_{j}\right\rangle=(2 / 2 i+1) \delta_{i j}$ & Uniform \\
Jacobi & $(1-x)^{a}(1+x)^{b}$ & {$[-1,1]$} & $\left\langle\psi_{i}, \psi_{j}\right\rangle=C_{\text {Jacobi }} \delta_{i j}$ & Beta \\
Laguerre & $\exp (-x)$ & {$[0,+\infty)$} & $\left\langle\psi_{i}, \psi_{j}\right\rangle=(\Gamma(i+1) / i !) \delta_{i j}$ & Exponential \\
General Laguerre & $\Gamma(x, a+1,1)$ & {$[0,+\infty)$} & $\left\langle\psi_{i}, \psi_{j}\right\rangle=\left(\begin{array}{c}i+a \\
i\end{array}\right) \delta_{i j}$ & Gamma \\
\hline
\end{tabular}

$$
\boldsymbol{\xi}=\left[\begin{array}{cccc}
\phi_{0}\left(\mathbf{x}^{(1)}\right) & \phi_{1}\left(\mathbf{x}^{(1)}\right) & \cdots & \phi_{n-1}\left(\mathbf{x}^{(1)}\right) \\
\phi_{0}\left(\mathbf{x}^{(2)}\right) & \phi_{1}\left(\mathbf{x}^{(2)}\right) & \cdots & \phi_{n-1}\left(\mathbf{x}^{(2)}\right) \\
\vdots & \vdots & \ddots & \vdots \\
\phi_{0}\left(\mathbf{x}^{(m)}\right) & \phi_{1}\left(\mathbf{x}^{(m)}\right) & \cdots & \phi_{n-1}\left(\mathbf{x}^{(m)}\right)
\end{array}\right]_{m \times n}
$$

Then, a regression model can be obtained as

$$
\widehat{Y}(u, v, w)=\boldsymbol{\Xi} \hat{a}(u, v, w)+\epsilon(u, v, w) .
$$

This is, an $n$-dimensional vector $\widehat{a}(u, v, w)=$ $\left[\widehat{a}_{0}(u, v, w), \widehat{a}_{1}(u, v, w), \ldots, \widehat{a}_{n-1}(u, v, w)\right]^{T}$ which represents the regression coefficients that are attached to explanatory variables $\quad \boldsymbol{\Xi}=\left[\phi_{0}(\mathbf{X}), \phi_{1}(\mathbf{X}), \ldots, \phi_{n-1}(\mathbf{X})\right]$, whereas the symbol $\epsilon(u, v, w)$ denotes residual errors:

$$
\epsilon(u, v, w)=\mathbf{y}(u, v, w)-\xi \widehat{a}(u, v, w),
$$

which consists of residual errors for all spatial and time realizations of the model. Following the theory of the multivariate regression, the unknown regression coefficients $\widehat{a}$ in equation (11) can be expressed in terms of the training data set $\xi$ and response sample $\mathbf{y}$ as

$$
\widehat{a}(u, v, w)=\left(\xi^{\mathrm{T}} \boldsymbol{\xi}\right)^{-1} \boldsymbol{\xi}^{\mathrm{T}} \mathbf{y}(u, v, w)
$$

Once the training matrix $\xi$ is defined in equation (10)and the corresponding aerodynamic response samples $\mathbf{y}$ are available, the minimization of the Euclidian-norm-based residual errors $\epsilon^{(i)}$ (with $i=1, \cdots, m$ ) allows finally deriving the regression-based surrogate model noted in equation (11), which will be useful to mimic the true but computationally intensive model $\eta(\cdot)$ in the afterwards uncertainty simulations.

\subsection{Uncertainty Analysis Based on the PCE Surrogate Model.} Once a surrogate model $\hat{\eta}(\mathbf{X} ; u, v, w)$ is obtained either analytically or numerically, the mean value and the variance of the aerodynamic response can be approximated as

$$
\left\{\begin{array}{l}
\mathrm{i} \dot{C} \check{L}[\eta(\mathbf{X} ; \mathrm{u}, \mathrm{v}, \mathrm{w})] \approx \mathrm{i} C^{\prime} \check{D}\left[\widehat{\mathrm{a}}_{0}(\mathrm{u}, \mathrm{v}, \mathrm{w})+\sum_{\mathrm{i}=1}^{\mathrm{n}-1} \widehat{\mathrm{a}}_{\mathrm{i}}(\mathrm{u}, \mathrm{v}, \mathrm{w}) \phi_{\mathrm{i}}(\mathbf{X})\right] \\
=\widehat{a}_{0}(u, v, w), \\
\operatorname{Var}[\eta(\mathbf{X} ; u, v, w)] \approx \operatorname{Var}\left[\widehat{a}_{0}(u, v, w)+\sum_{i=1}^{n-1} \widehat{a}_{i}(u, v, w) \phi_{k}(\mathbf{X})\right] \\
=\sum_{i=1}^{n-1} \widehat{a}_{i}^{2}(u, v, w) .
\end{array}\right.
$$

Besides, the Sobol' index used for the variance-based global sensitivity analysis can be easily expressed by means of the PCE coefficients [42]. To implement, an index set of representing the $i$ th variance component $V_{i}$ uniquely contributed by the random variable $X_{i}$ is first defined as

$$
\{\boldsymbol{\beta}\}_{i}=\arg \left\{\boldsymbol{\alpha}: \bigcup_{|\boldsymbol{\alpha}| \leq p}\left\{\left[\alpha_{i} \neq 0\right] \cap\left[\alpha_{j}=0\right]_{j=1, j \neq i}^{d}\right\}\right\} .
$$

Similarly, a $q$-dimensional index set $\mathbf{q}=\left\{i_{1}, \cdots, i_{q}\right\}$ that measures the joint variance effect due to multiple input random variables $\left\{X_{i_{1}}, \cdots, X_{i_{q}}\right\}$ acting together is presented as

$$
\{\boldsymbol{\beta}\}_{\mathbf{q}}=\arg \left\{\boldsymbol{\alpha}: \bigcup_{|\boldsymbol{\alpha}| \leq p}\left\{\left[\alpha_{k} \neq 0\right], \forall k \in q\right\} \cap\left\{\left[\alpha_{k}=0\right], \forall k \notin q\right\}\right\} .
$$

Based on abovementioned notations, results for the main and the joint Sobol' sensitivity indices for the variance-based global sensitivity analysis can be readily expressed as

$$
\begin{gathered}
S_{i}(u, v, w)=\frac{\sum_{\boldsymbol{\alpha} \in\{\boldsymbol{\beta}\}_{i}} a_{\boldsymbol{\alpha}}^{2}(u, v, w)}{\sum_{k=1}^{n-1} a_{k}^{2}(u, v, w)}, \quad i=1,2, \ldots, d, \\
S_{\mathbf{q}}(u, v, w)=\frac{\sum_{\boldsymbol{\alpha} \in\{\boldsymbol{\beta}\}_{\mathbf{q}} a_{\boldsymbol{\alpha}}^{2}(u, v, w)}}{\sum_{k=1}^{n-1} a_{k}^{2}(u, v, w)}, \quad 1 \leq i_{1}, \ldots, i_{q} \leq d .
\end{gathered}
$$

Note that the parameter $a_{\boldsymbol{\alpha}}(u, v, w)$ denotes the PCE coefficient attached to a $d$-dimensional polynomial $\phi_{\boldsymbol{a}}(\mathbf{X})$. Therefore, results for Sobol' sensitivity index are purely expressed in terms of the PCE coefficients.

To summarize, the proposed approach for uncertain aerodynamic analysis of an airfoil includes (1) the development of the chaos polynomial set $\left\{\phi_{i}(\mathbf{X})\right\}_{i=0}^{n-1}$ in equations (1) and (2) the calculation of PCE coefficients by using the statistical regression method in equations (3) and (13) the sensitivity analysis and the response distribution estimation in Section 2.4. Therefore, in conjunction with a small number of low-discrepancy simulations and a simple postprocessing procedure the uncertainty analysis of an airfoil model can be effectively realized via the polynomial chaos expansion method.

\section{Uncertainty Analysis of the Helmholtz Equation}

This section illustrates an application of the polynomial chaos expansion approach for the uncertainty analysis of the two-dimensional Helmholtz equation, which is generally defined as 


$$
\left\{\begin{array}{l}
-\left(\frac{y^{2}(u, v)}{\partial u^{2}}+\frac{y^{2}(u, v)}{\partial v^{2}}\right)+\omega(u, v) y(u, v)=g_{0}(u, v), \\
\Omega \in[a, b] \times[c, d] \\
\left.y(u, v)\right|_{\Gamma_{1}}=g_{1}(u, v) \\
\left.\frac{\partial y(u, v)}{\partial \mathbf{n}}\right|_{\Gamma_{2}}=g_{2}(u, v)
\end{array}\right.
$$

where $\Gamma_{1}$ represents the Dirichlet boundary condition and $\Gamma_{2}$ denotes the Neumann boundary condition. For uncertainty analysis, the wave-number function $\omega(u, v ; \theta)$ is molded by using the Gaussian random field. This implies the response quantity of this equation is spatially stochastic as $u, v \in[a, b] \times[c, d]$.

3.1. The Karhunen-Loève Expansion for Spatial Variabilities. According to the theory of a random field simulation, the spatially varying two-dimensional random property $\omega(u, v ; \boldsymbol{\theta})$ can be effectively represented by means of the Karhunen-Loève (K-L) expansion [43] and its variants [44]:

$$
H(u, v ; \boldsymbol{\theta})=\mu(u, v)+\sum_{i=1}^{\infty} \sqrt{\lambda_{i}} X_{i}(\boldsymbol{\theta}) \phi_{i}(u, v) .
$$

Herein, $\mu(u, v)$ denotes the mean value of the random field, whereas the statistically Gaussian random variables $X_{i}(\theta)$ in this example can be obtained by

$$
X_{i}(\theta)=\frac{1}{\sqrt{\lambda_{i}}} \int_{\Omega} \int_{\Omega}[H(u, v ; \boldsymbol{\theta})-\mu(u, v ; \boldsymbol{\theta})] \psi_{i}(u, v) \mathrm{d} u \mathrm{~d} v,
$$

where $\lambda_{i}$ and $\phi_{i}(u, v)$ denote eigenvalues and eigenfunctions for the covariance function $\operatorname{Cov}(\mathbf{u}, \mathbf{v})$, respectively, which are solutions of the following integral eigenvalue problem (IEVP):

$$
\int_{\Omega} \int_{\Omega} \operatorname{Cov}(\mathbf{u}, \mathbf{v}) \psi_{i}\left(u^{\prime}, v^{\prime}\right) \mathrm{d} u^{\prime} \mathrm{d} v^{\prime}=\lambda_{i} \psi_{i}(u, v) .
$$

Once a numerical solution of the integral integration is available, numerical realization of the random field based on an $m$-term $\mathrm{K}$ - $\mathrm{L}$ expansion is given as

$$
\widetilde{H}(u, v ; \boldsymbol{\theta})=\mu(u, v)+\sum_{i=1}^{M} \sqrt{\lambda_{i}} \psi_{i}(u, v) X_{i}(\boldsymbol{\theta}) .
$$

In numerical simulations, the following exponential model is assumed for the spatially varying wave-number function:

$$
\operatorname{Cov}(\mathbf{u}, \mathbf{v})=\sigma_{0}^{2} \exp \left(-\frac{\left|\mathbf{u}-\mathbf{u}^{\prime}\right|}{\delta_{\mathbf{u}}}-\frac{\left|\mathbf{v}-\mathbf{v}^{\prime}\right|}{\delta_{\mathbf{v}}}\right), \quad \text { as } \mathbf{u}, \mathbf{v} \in \Omega .
$$

Figure 1 presents simulation results for random field of the wave-number function $\omega(u, v ; \boldsymbol{\theta})$ based on $10^{4}$ random samples. Herein, parameters in the covariance function are given as $\sigma_{0}=\pi, \delta_{u}=0.8$, and $\delta_{v}=1$. With the truncation parameter $M=5$, the error for the variance simulation result in Figure 1(c) is determined as $4.15 \%$, which implies the simulation variance is relatively lower than that of the benchmark variance $\pi$.

3.2. Numerical Results for the Uncertainty Analysis. With numerical simulation results for random field of the wavenumber function, the procedure summarized in Section 2.4 is used for uncertainty analysis of the Helmholtz equation. The multivariate Hermit polynomials are first determined to represent the Gaussian random variables produced by the K-L expansion, and the PCE model for stochastic response of the Helmholtz equation is determined as

$$
\widehat{\eta}(\mathbf{X} ; u, v)=\sum_{k=0}^{n-1} a_{k}(u, v) \phi_{k}(\mathbf{X}),
$$

where the random vector is defined as $\mathbf{X}=\left[X_{1}, \ldots, X_{5}\right]^{T}$ and $X_{i}$ is the standard Normal random variable determined by the K-L expansion in equation (20).

Assume that the highest order of the chaotic polynomial is $p=6$. This implies the total number of polynomial terms in equation (24) will be 462 . If the Gaussian quadrature method used to evaluate the PCE coefficients, it involves $7,776\left(=6^{5}\right)$ functional evaluations in total. Herein, each random variable is represented by six Gaussian-Hermit nodes. To reduce the computational cost for multivariate cases, the statistical regression method in Section 2.3 with 500 samples were alternatively used, and the corresponding error term is determined to evaluate numerical accuracy of this PCE model:

$$
\varepsilon^{2}(u, v)=\frac{\sum_{i=1}^{N_{\mathrm{MCS}}}\left[\eta\left(\mathbf{x}^{(i)} ; u, v\right)-\widehat{\eta}\left(\mathbf{x}^{(i)} ; u, v\right)\right]^{2 N_{\mathrm{MCS}}}}{\sum}\left[\eta\left(\mathbf{x}^{(i)} ; u, v\right)-\bar{Y}(u, v)\right]^{2},
$$

where $\eta(\cdot)$ and $\hat{\eta}(\cdot)$ represent the simulated and the predicted model responses, respectively, whereas $\bar{Y}(u, v)$ denotes the mean value of the model response, i.e.,
$\bar{Y}(u, v)=\left(1 / N_{\mathrm{MCS}}\right) \sum_{i=1}^{N_{\mathrm{MCS}}} \eta\left(\mathbf{x}^{(i)} ; u, v\right)$. Therefore, an indicator $R^{2}$ is defined as $R^{2}(u, v)=1-\varepsilon^{2}(u, v)$ in the following simulations to quantify numerical accuracy of the PCE model. 

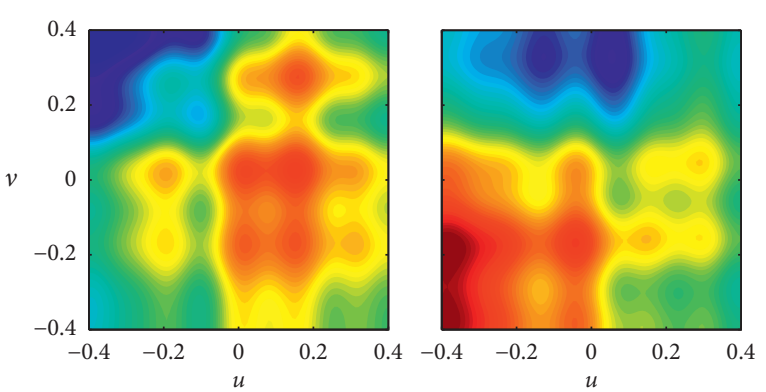

(a)

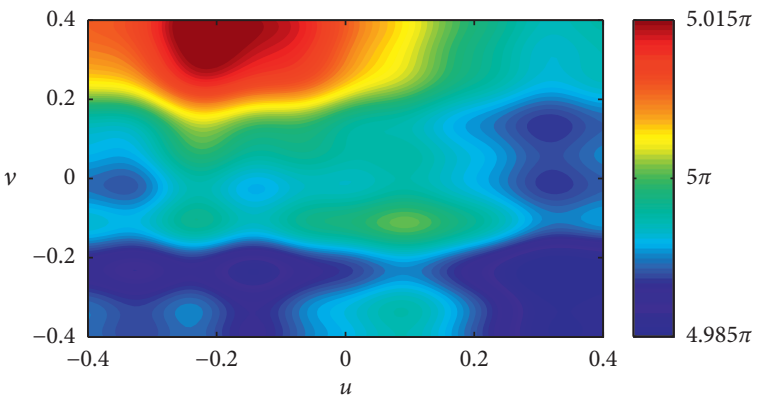

(b)
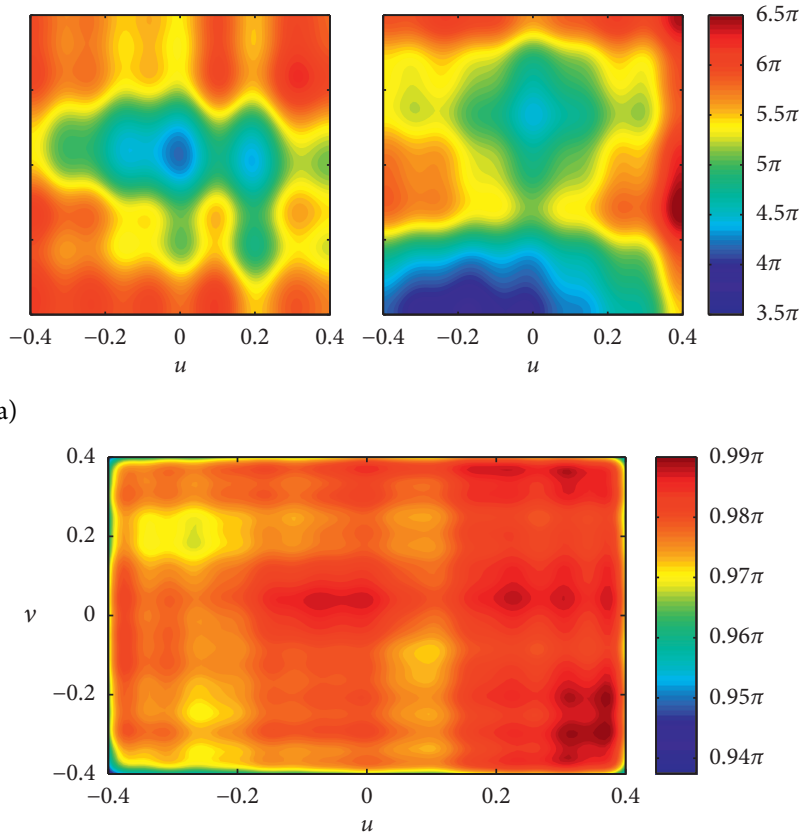

(c)

Figure 1: Results for Monte Carlo simulation of the two-dimensional random field in terms of the wave-number function (mean-value function $\mu(u, v)=5 \pi$, and the sample size is $\left.10^{4}\right)$. (a) Four realizations for random field of the wave-number function $\omega(u, v, \theta)$. (b) Mean value. (c) Standard deviation.

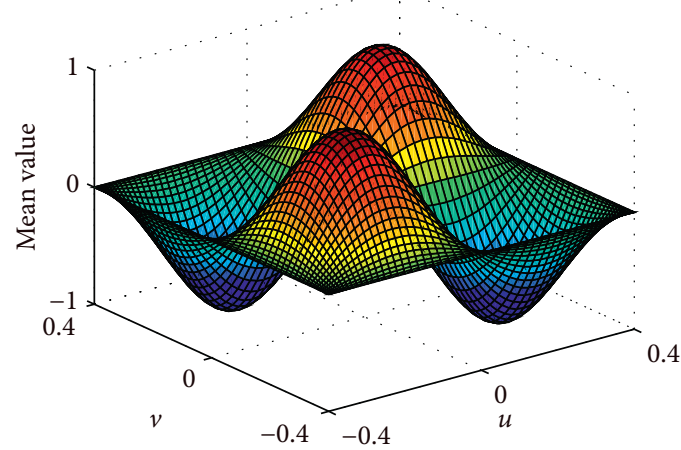

(a)

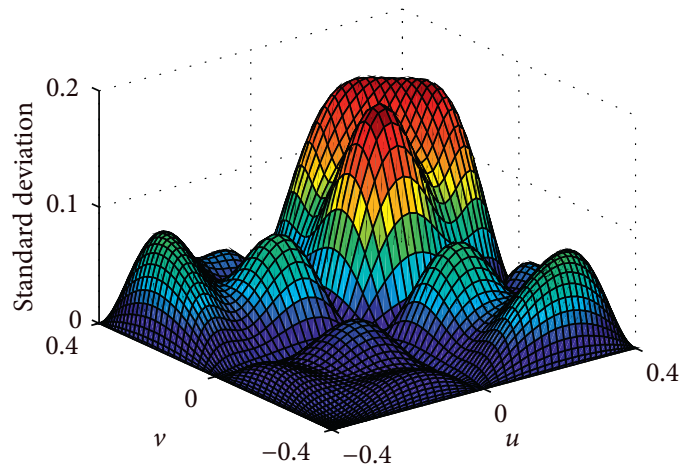

(c)

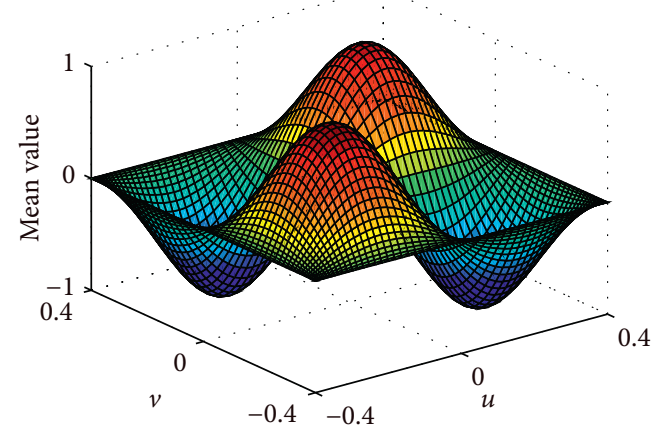

(b)

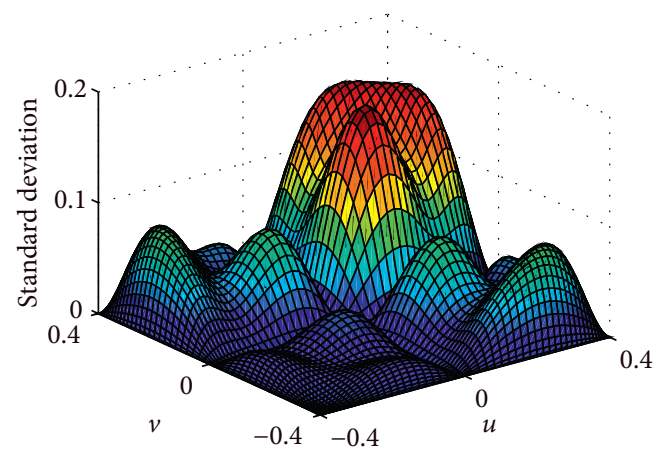

(d)

FIGURE 2: Mean value and standard deviation for stochastic response of the Helmholtz equation. (a) Mean value: brutal-force simulation. (b) Mean value: the PCE method. (c) Standard deviation: brutal-force simulation. (d) Standard deviation: the PCE method. 


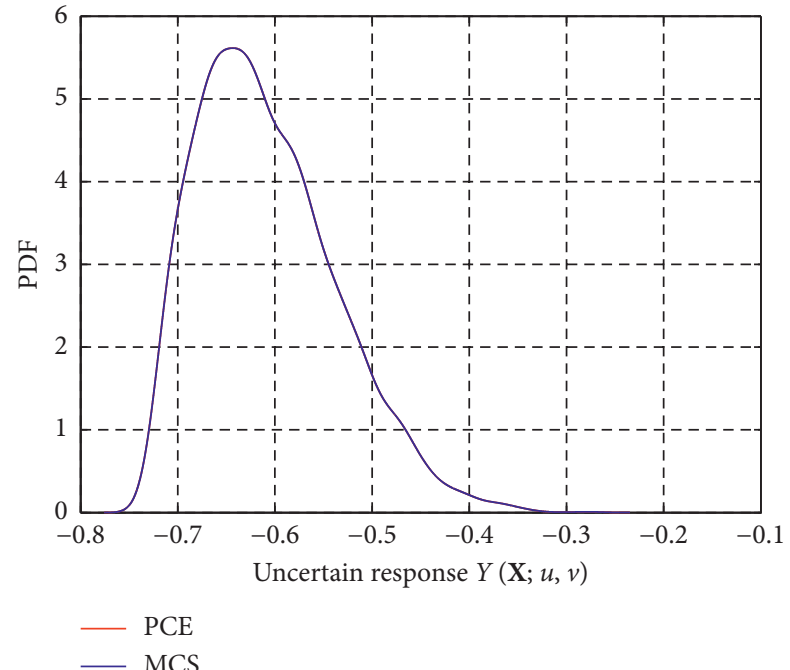

(a)

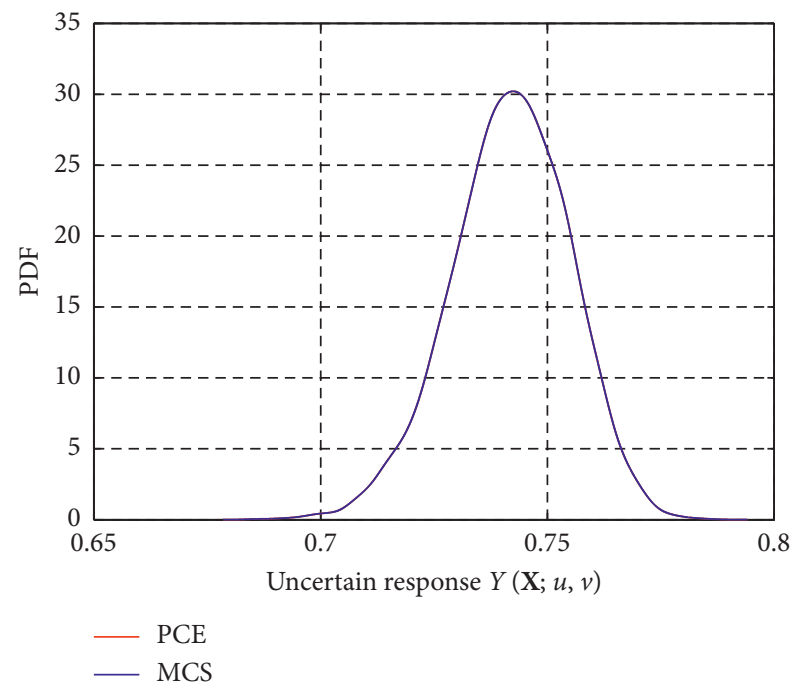

(c)

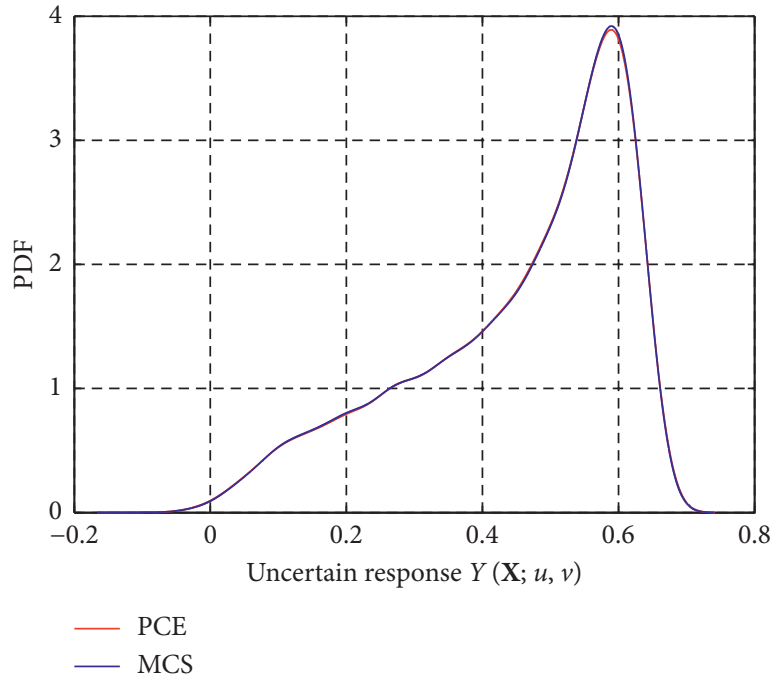

(b)

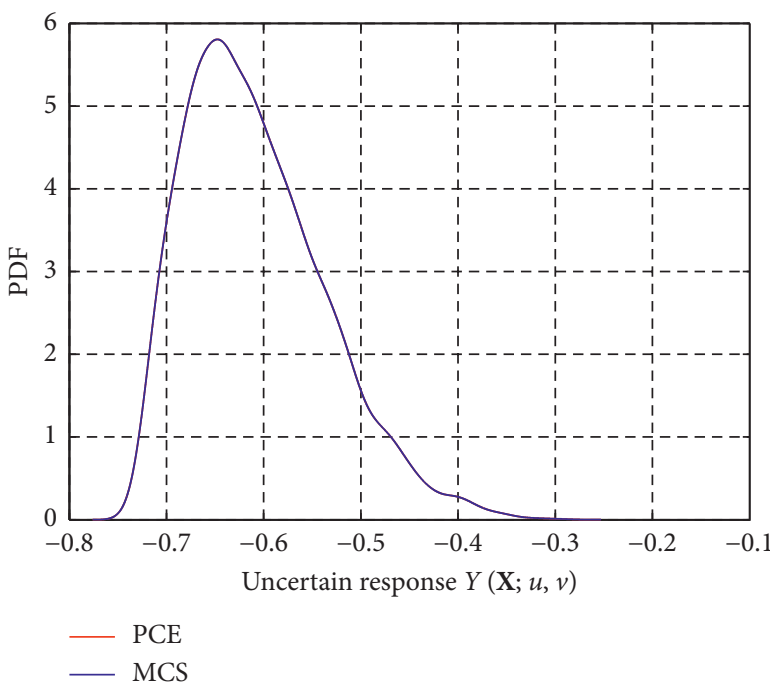

(d)

FIgUre 3: Verifications for output probability distribution of the Helmholtz equation. (a) Position A $(-0.268,0.297)$. (b) Position B (-0.297, 0.297). (c) Position C (-0.268, 0.268). (d) Position D (-0.297, 0.268).

Figure 2 depicts results for the mean value and the standard deviation for stochastic responses of the Helmholtz equation. With the spatially varying random property for the wavenumber function $\omega(u, v ; \theta)$, results determined by the brutalforce MCS with $10^{4}$ samples are provided for numerical verifications. The proposed regression algorithm with 500 lowdiscrepancy training samples is able to determine accurate simulation results for the PCE model. The maximal fitting error result is determined as $\max _{u, v \in \Omega}\left\{\varepsilon^{2}(u, v)\right\}=1.355 \times 10^{-3}$. The small regression error has verified numerical accuracy of this approach in developing the PCE model for uncertainty analysis of the Helmholtz equation.

Given the PCE model for the system response, Figure 3 further presents simulation results of $Y(\mathbf{X} ; u, v)$ for some locations within the simulation domain $\Omega=[-0.4,0.4] \times$ $[-0.4,0.4]$. Note that $10^{4}$ samples were used to determine the benchmark result. A close agreement of the simulation and the predicted results has confirmed the effectiveness of this PCE model for uncertain analysis of the Helmholtz equation with spatially varying wave-number property.

\section{Uncertain Aerodynamic Analysis of the NACA 63-215 Airfoil}

This section considers uncertain aerodynamic analysis of the NACA 63-215 airfoil via the polynomial chaos expansion approach. To achieve this, the deterministic aerodynamic simulation is first realized based on a finite element model in conjunction with the Spalart-Allmaras (S-A) turbulence model. In this simulation, the angle of attack was considered from $-4^{\circ}$ to $18^{\circ}$ with an incremental step $0.5^{\circ}$.

Together with results provided by the wind tunnel test [45] and XFoil computational package [46], the applicability of the simulation model is first verified. Note that the 


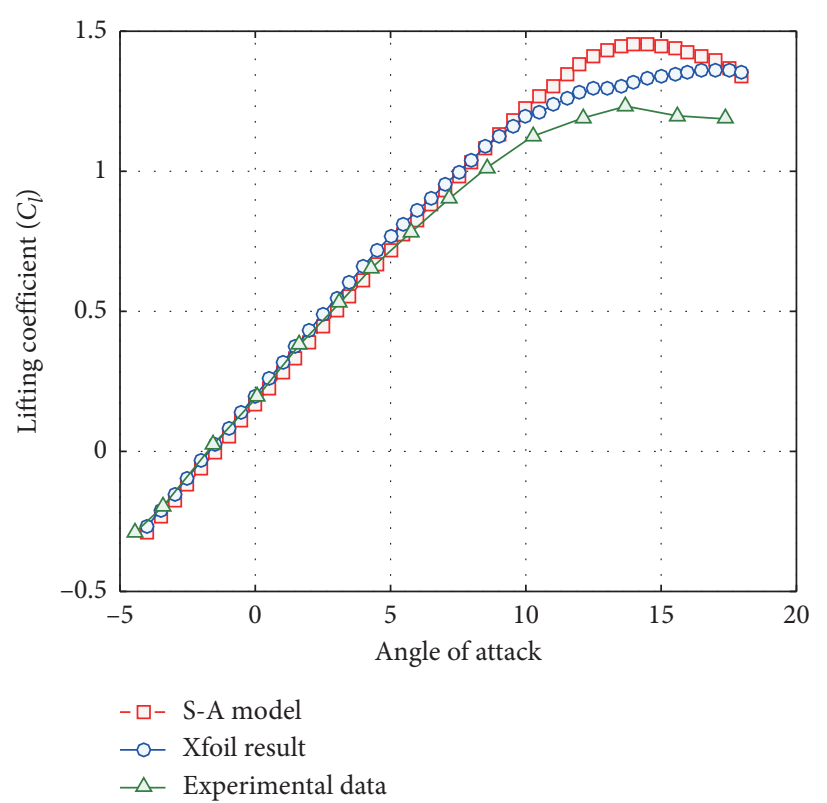

(a)

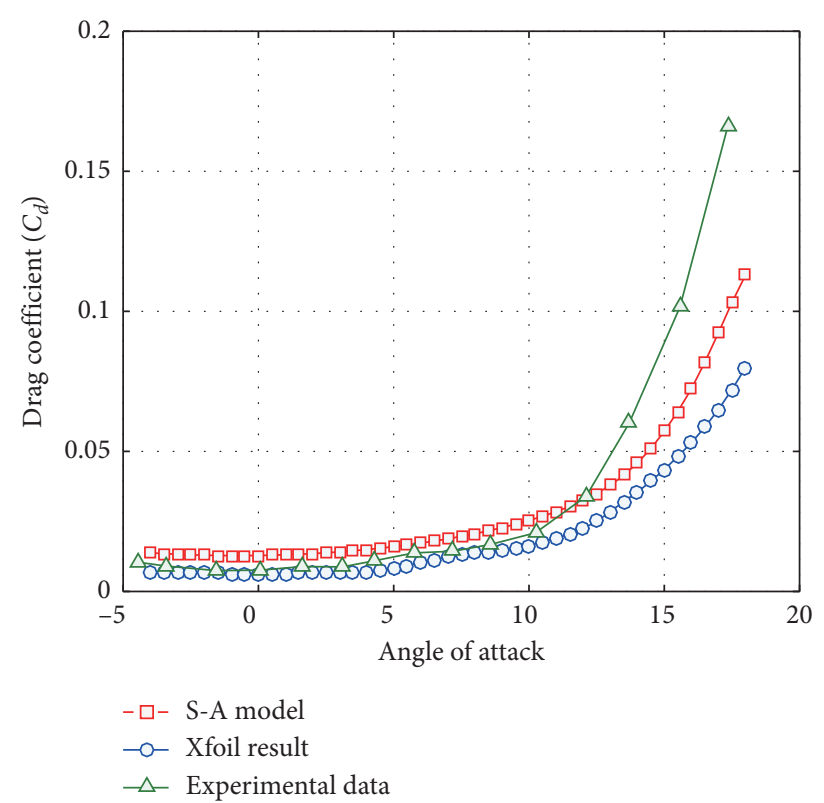

(b)

FIGURE 4: Results for aerodynamic characteristic of the NACA 63-215 airfoil determined by various turbulence models. (a) Coefficients of lift. (b) Coefficients of drag.

following simulation parameters are considered: the wind velocity $V=16 \mathrm{~m} / \mathrm{s}$, the air density $\rho=1.225 \mathrm{~kg} / \mathrm{m}^{3}$, and the viscosity parameter $1.7894 \times 10^{-5} \mathrm{~kg} / \mathrm{m} \cdot \mathrm{s}$.

Figure 4 presents the simulation results for the lifting and the drag coefficients of this airfoil. It is observed that results for the lift coefficient provided by the FEM with the S-A turbulence model are closely agreed with the results provide by the XFoil and the experimental data. Compared with the wind tunnel test data, the drag coefficient result predicted by the S-A is more accurate than that of the XFoil. Therefore, the uncertain aerodynamic quantification of the NACA 63-215 airfoil is implemented based on the S-A model for the pressure and the velocity response fields. Once a numerical simulation model of the airfoil is available, the corresponding uncertainty analysis can be realized via the polynomial chaos expansion approach. Note that the maximal lift-drag ratio is determined as $A O A^{*}=9.5^{\circ}$ in this deterministic simulation, and the PCE approach is further applied to provide estimation results for the probability distribution of the drag, the lift, and the life-to-drag ratio at this AOA value as follows.

4.1. Results for the Uncertainty Analysis. To implement the uncertain aerodynamic analysis, the probabilistic characteristics of input random factors are listed in Table 2. This includes the inflow velocity $V_{\infty}$, the air density $\rho$, and the viscosity parameter $v$. The PCE algorithm is realized for the uncertain analysis, and the brutal-force Monte Carlo simulation with $10^{4}$ samples is assumed to provide the benchmark result for the numerical verification.

At first, the PCE models for the coefficient of pressure $C p\left(\mathbf{X} ; u^{\prime}, v^{\prime}\right)$ and the pressure response of the simulation field are separately developed:
TABLE 2: Random variables for uncertainty aerodynamic analysis of the NACA 63-215 airfoil.

\begin{tabular}{lcccr}
\hline Random variables & Symbol & Distribution & Mean & COV \\
\hline Wind velocity $(\mathrm{m} / \mathrm{s})$ & $V_{\infty}$ & Gaussian & 20 & 0.05 \\
Air density $\left(\mathrm{kg} / \mathrm{m}^{3}\right)$ & $\rho$ & Gaussian & 1.225 & 0.05 \\
$\begin{array}{l}\text { Viscosity parameter } \\
(\mathrm{cP})\end{array}$ & $v$ & Gaussian & $1.7894 \times 10^{-2}$ & 0.05 \\
\hline
\end{tabular}

$$
\left\{\begin{array}{l}
\widehat{C} p(\mathbf{X} ; u, v)=\sum_{k=0}^{d-1} a_{C p k}(u, v) \phi_{k}(\mathbf{X}), \\
\widehat{P}(\mathbf{X} ; u, v)=\sum_{k=0}^{d-1} a_{P k}(u, v) \phi_{k}(\mathbf{X})
\end{array}\right.
$$

in which the chaotic polynomial terms $\phi_{k}(\mathbf{X})$ are expressed as the tensor product of the Hermit polynomials to represent normal random variables in Table 2. With 500 samples generated based on the LSH approach, the statistical regression method in Section 2.3 is used to determine the PCE coefficient.

In this PCE model, the polynomial order parameter is given as $p=3$. This determines totally 20 terms for numerical representation of the uncertain aerodynamic response. Following the moment results based on the PCE coefficients in equation (14), the first-two orders of moments for the aerodynamic responses are presented in Figure 5. A close agreement between the simulation and the predicted moment results has confirmed the high accuracy of this PCE method for the pressure coefficient $C_{p}$ of this airfoil.

Results for the mean value and the standard deviation of this pressure response field are determined, as shown 


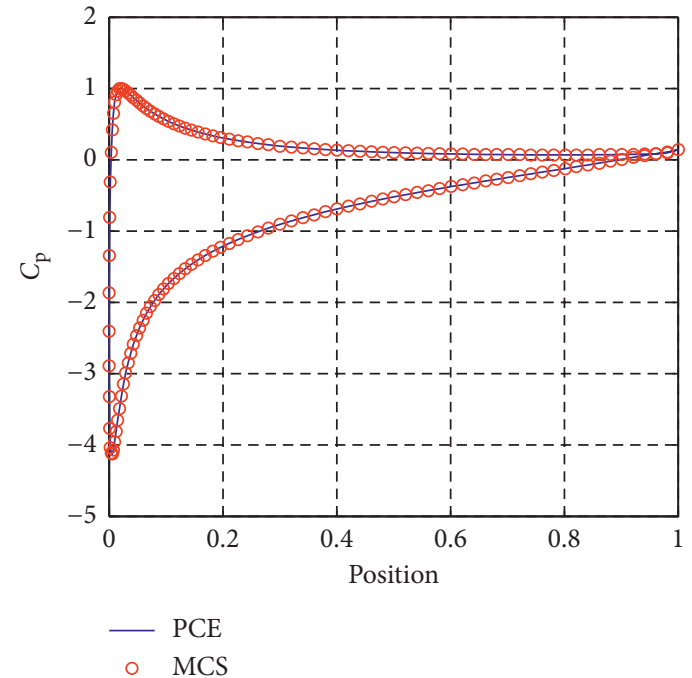

(a)

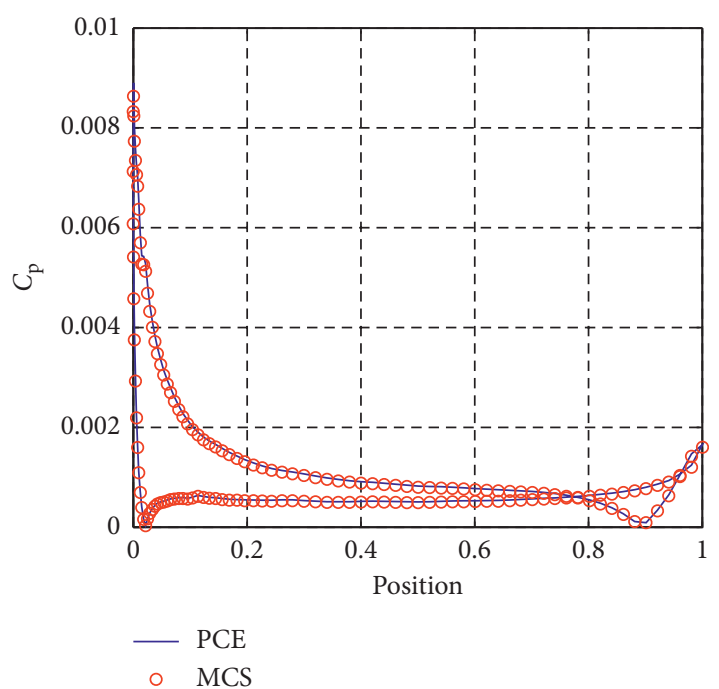

(b)

Figure 5: Numerical results for the mean and the standard deviation of $C_{p}$ at the airfoil surface. (a) The mean value. (b) The standard deviation.

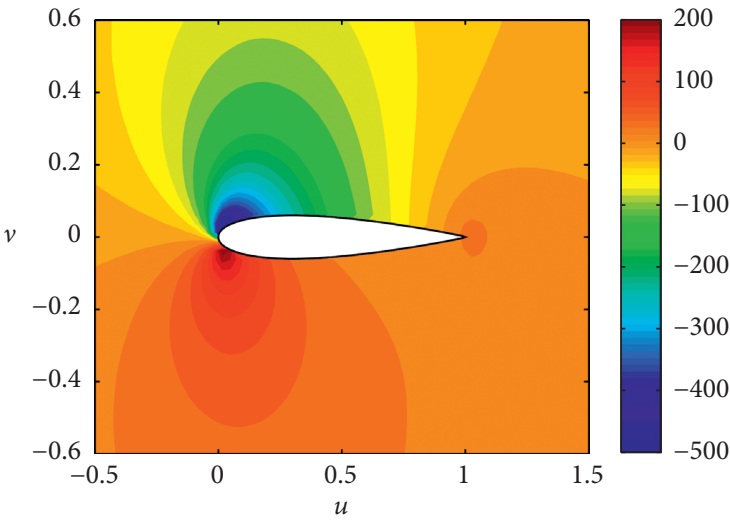

(a)

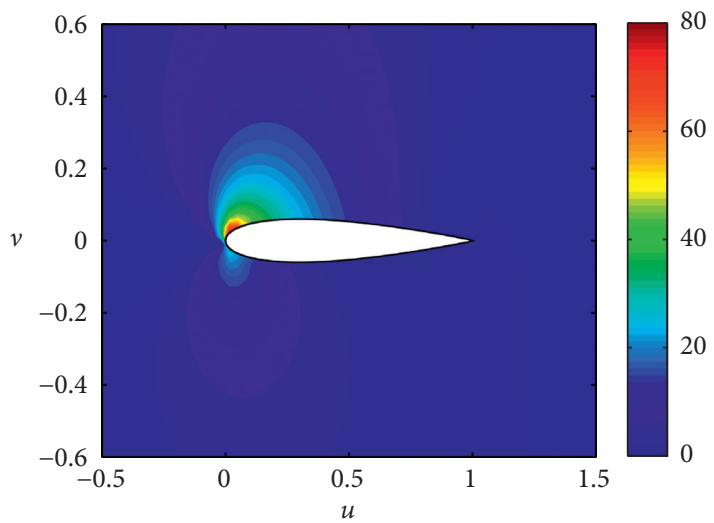

(c)

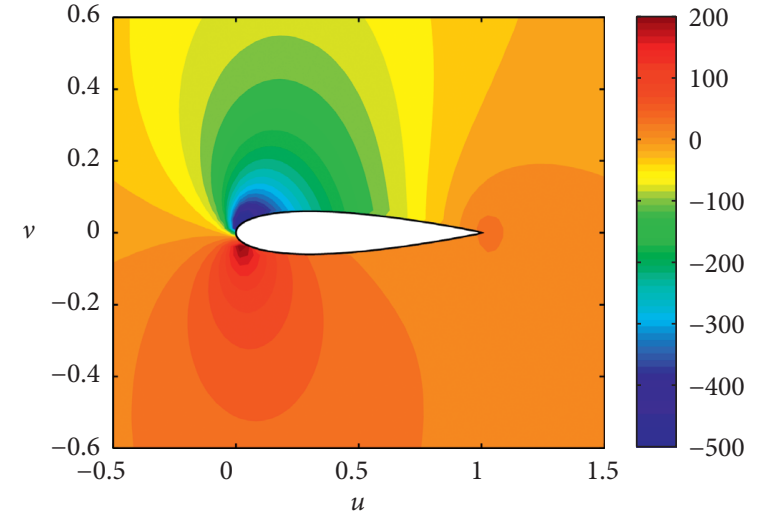

(b)

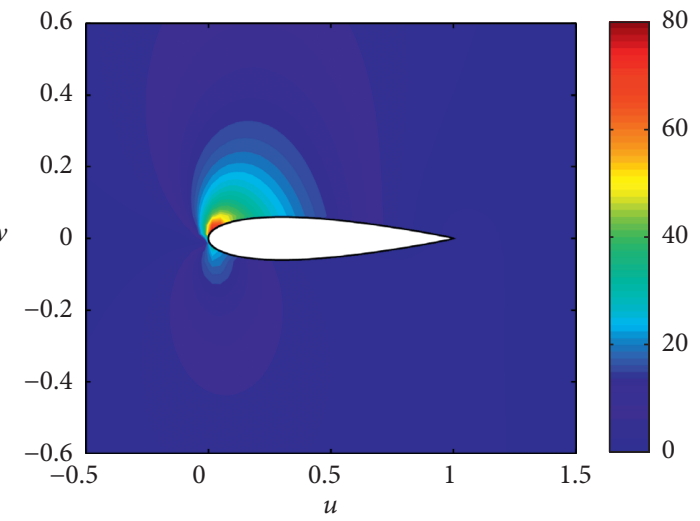

(d)

Figure 6: Results for the mean value and the standard deviation of the pressure response field. (a) Mean value: the Monte Carlo simulation. (b) Mean value: the PCE method. (c) Standard deviation: the MCS method. (d) Standard deviation: the PCE method. 


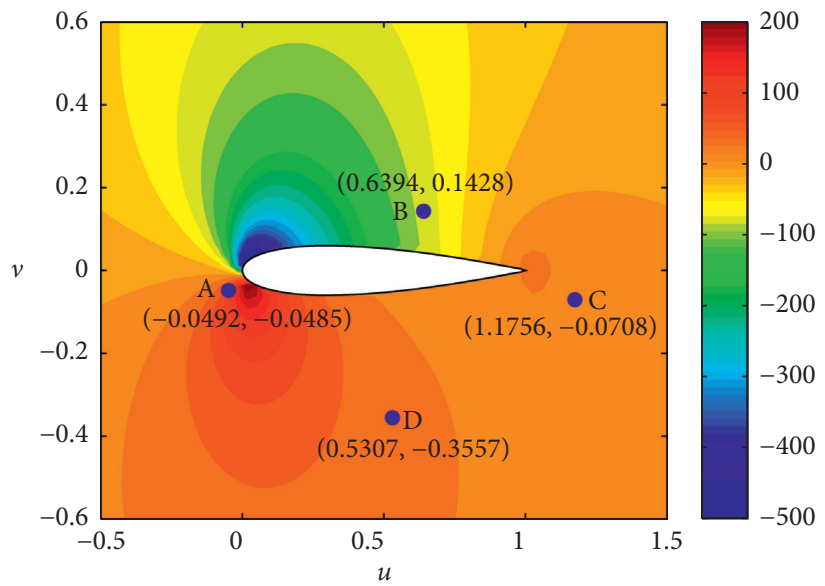

FIGURE 7: Reference positions for uncertainty analysis of the response pressure field.
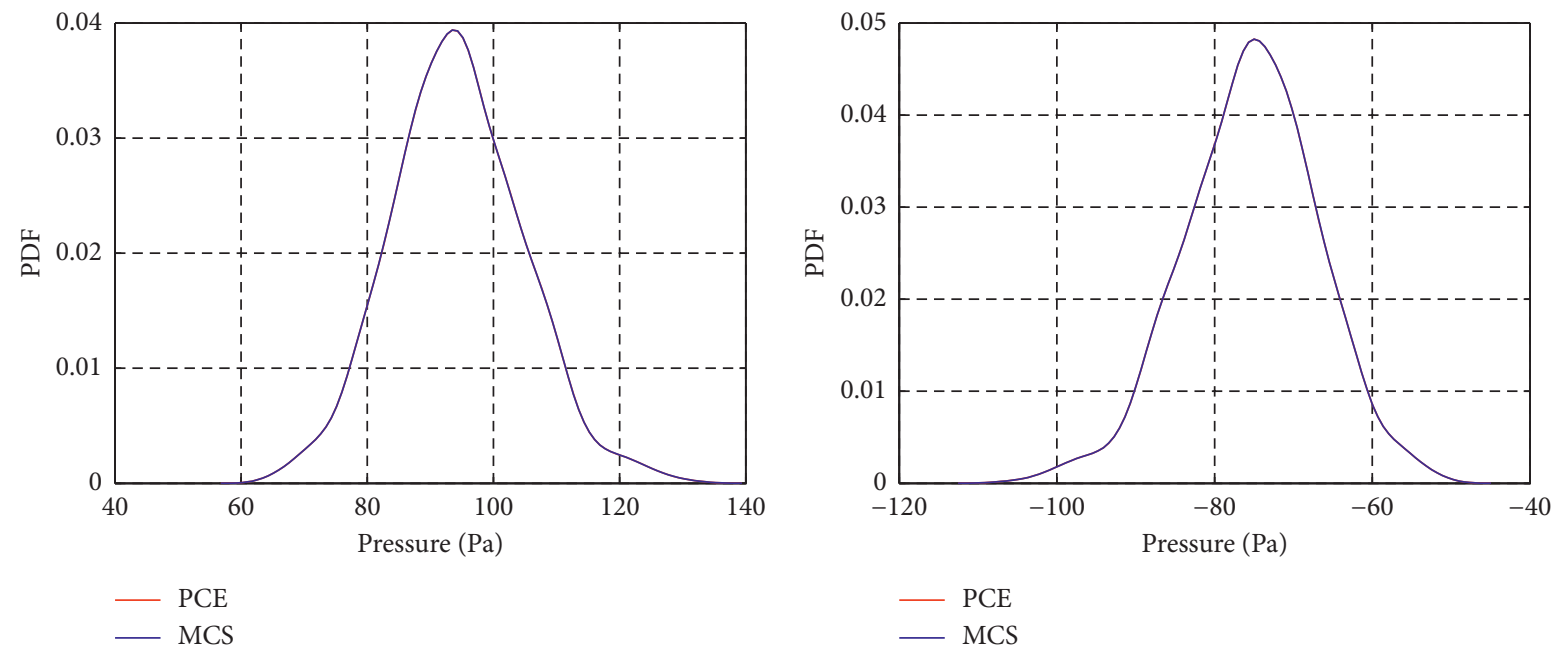

(a)
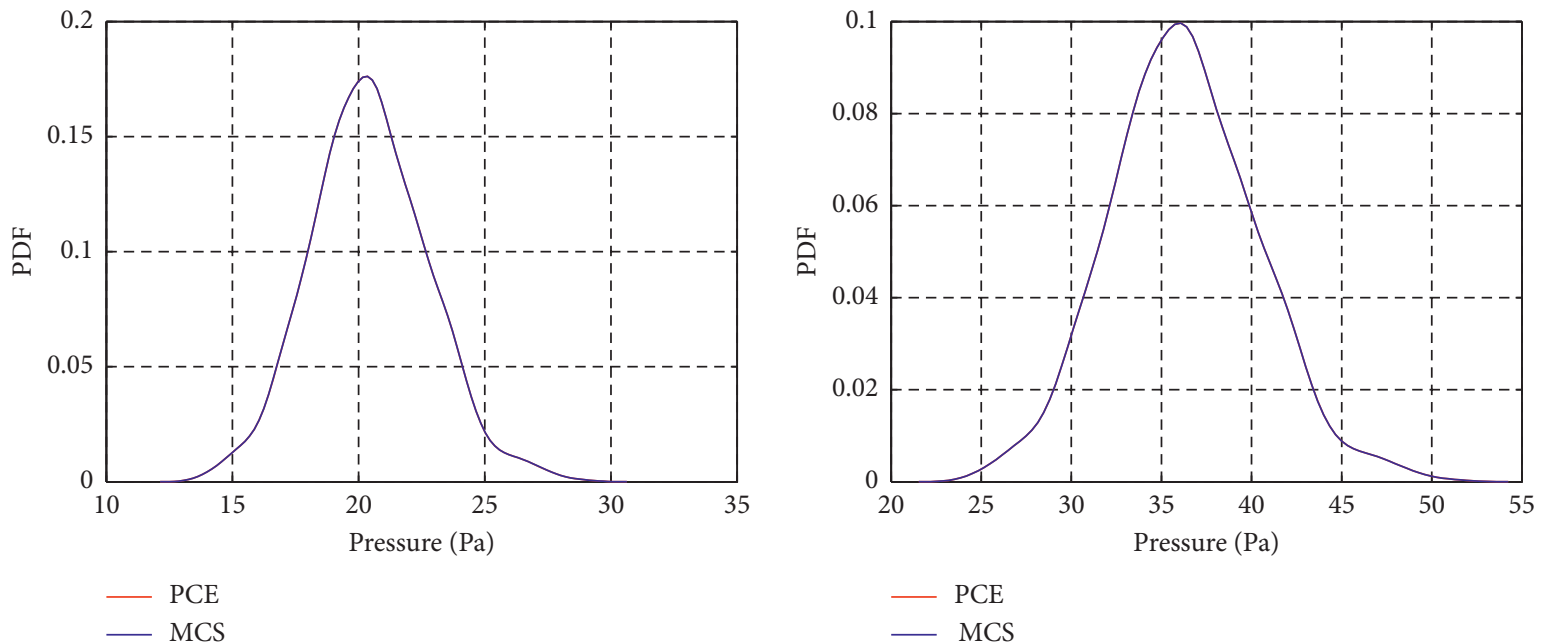

(c)

(d)

Figure 8: Results for probability distribution of the pressure response at four positions. (a) Position A: $(-0.0492,-0.0485)$. (b) Position B: (0.6394, 0.1428). (c) Position C: $(1.1756,-0.0708)$. (d) Position D: $(0.5307,-0.3557)$. 


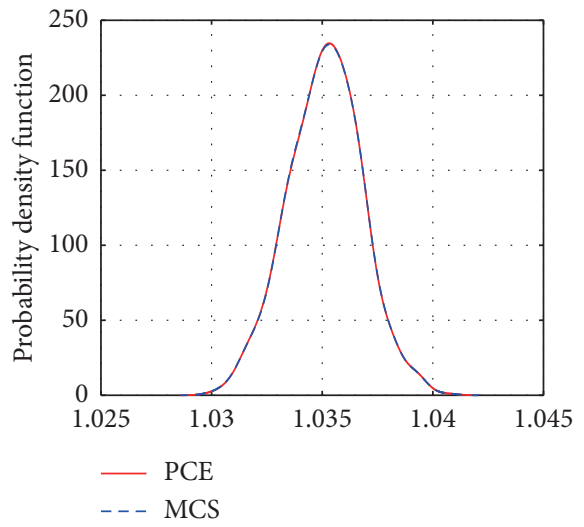

(a)

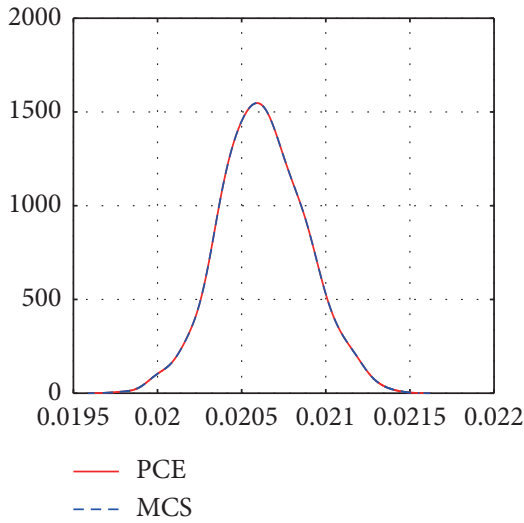

(b)

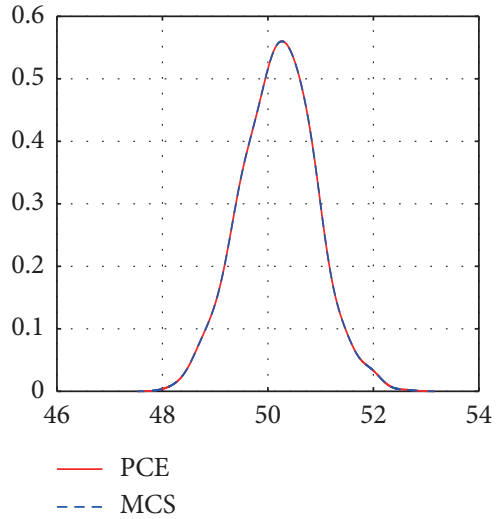

(c)

FIGURE 9: Results for probability distribution of (a) the lift coefficient, (b) the drag coefficient, and (c) the ratio of lift-to-drag.

in Figure 6. Note that results for all simulation points within the investigated domain were determined. The regression parameters $a_{k}(u, v)$ are spatially dependent. Combined with benchmark result provided based on $10^{4}$ samples, the proposed approach is fairly efficient for numerical estimation of the moment of the response random filed.

In this surrogate model, deterministic simulations were numerically realized based on a personal computer with the CPU of Interl $(R)$ Core (TM) $i 7-37703.40 \mathrm{GHz}$ and the $4 \mathrm{~GB}$ physical memories. In addition, four-thread parallel computing technique was implemented to accelerate the simulation process. Each round of the deterministic simulation approximately needs 3 seconds. Therefore, with 500 samples to develop the PCE surrogate model, the total simulation time is around three minutes in total. However, the brutalforce Monte Carlo simulation with $10^{4}$ samples requires 9.5 hours. This has demonstrated the high efficiency of this proposed approach for uncertain aerodynamic analysis of the NACA 63-215 airfoil.

Numerical verification of the PCE approach is further extended to the probability distribution for pressure responses at four locations, as shown in Figure 7 . This includes the point $A(-0.0492,-0.0485)$, the point $B(0.6394,0.1428)$, the point $C(1.1756,-0.0708)$, and the point $D(0.5307,-0.3557)$. Results for the probability distribution of the pressure are summarized in Figure 8. Note that results for the accuracy measure in equation (25) are generally less than $6.0 \times 10^{-8}$. Together with accuracy simulation results for probability distribution of the lift and drag coefficients in Figure 9 for the case of $A O A^{*}=9.5^{\circ}$, it has confirmed the effectiveness of utilizing this polynomial chaos expansion for stochastic aerodynamic analysis of airfoils.

4.2. Results for the Global Sensitivity Analysis. The section further determines results for the global sensitivity analysis of the NACA 63-215 airfoil with random variables in terms of the inflow velocity $V_{\infty}$, the air density $\rho$, and the dynamic viscosity. To achieve this, surrogate models for $C_{l}, C_{d}$, and $C_{l d}$ are separately determined. In this regard,
TABLE 3: Results for sensitivity analysis of the airfoil model.

\begin{tabular}{lccc}
\hline Sensitivity & $C_{l}$ & $C_{d}$ & $C_{l d}$ \\
\hline$S_{1}$ & 0.3901 & 0.4221 & 0.3942 \\
$S_{T_{1}}$ & 0.3929 & 0.4324 & 0.4032 \\
$S_{2}$ & 0.3454 & 0.3237 & 0.3457 \\
$S_{T_{2}}$ & 0.3536 & 0.3339 & 0.3504 \\
$S_{3}$ & 0.2645 & 0.2543 & 0.2601 \\
$S_{T_{3}}$ & 0.2851 & 0.2946 & 0.2735 \\
\hline
\end{tabular}

the regression error are determined as $3.14 \times 10^{-6}$, $3.26 \times 10^{-7}$, and $2.28 \times 10^{-7}$ for $C_{l}, C_{d}$, and $C_{l d}$, respectively. The small regression errors $\left(\leq 10^{-5}\right)$ for all PCE models have confirmed the high accuracy of this proposed approach.

With the polynomial order parameter $p=3$, the chaotic Hermit polynomials are used to develop the PCE surrogate model for aerodynamic response of the NACA 63-215 airfoil. The statistical regression method with 500 Sobol' samples was used to determine the expansion coefficient. Besides, the brutal-force Monte Carlo simulation with $10^{4}$ samples was used to provide benchmark results for this global sensitivity analysis.

Based on the PCE coefficients determined with the statistical regression method, Table 3 summarizes results for the global sensitivity analysis of this uncertain aerodynamic model with random variables in terms of the $V_{\infty}$, the $\rho$, and the dynamic viscosity parameter $\nu$. It is observed that these three random factors have almost contributed equally for total aerodynamic variance of $C_{l}$, $C_{d}$, and $C_{l d}$, whereas the parameter of the wind velocity $V_{\infty}$ has been ranked as the principle factor given the largest value of the global sensitivity result. This is followed by the air density $\rho$ and the dynamic viscosity parameter $\nu$. Specially, results for the total sensitivity index $S_{T_{i}}$ are slightly larger than those of the primary sensitivity index $S_{i}$, given that the total variance includes joint variance contributions of $X_{i}$ and $X_{j}$ together. To summarize, the proposed PCE surrogate model can provide reliable estimation results for the global sensitivity analysis of the NACA 63-215 airfoil. 


\section{Conclusions}

The paper presents an effective approach for uncertain aerodynamic analysis of airfoils via the polynomial chaos expansion. To archive this, the spatially varied random properties of a partial differential equation are first resented as the normal random variables based on the K-L expansion method. Then, a set of basis functions are determined by using the chaotic orthogonal polynomials. This allows to developing a statistical regression model for the uncertain aerodynamic analysis via the polynomial chaos expansion approach. In numerical simulations, the Helmholtz equation with a spatially varied wave-number function is considered. With the truncated K-L expansion scheme to represent the input random field, the polynomial chaos expansion method is able to provide reliable estimation results for the statistical moment and the probability distribution of the model response. This verification is further extended to the uncertain aerodynamic analysis of the NACA 63-215 airfoil, which is modelled by using the normal random variables. The deterministic aerodynamic simulation is realized by using the finite element method in conjunction with the Spalart-Allmaras turbulence model. With a small number of deterministic model evaluations, results for the response pressure and velocity fields of this airfoil are determined. Compared to benchmark results provided by the brutalforce Monte Carlo simulation, it has confirmed the high accuracy of this method for the global sensitivity analysis of aerodynamic models. The close agreement between the simulation and the predicted results for the lift, the drag, and the lift-to-drag ratio has confirmed the effectiveness of this approach for uncertain aerodynamic analysis of airfoils.

\section{Data Availability}

The simulation data within this submission are available based on the request.

\section{Conflicts of Interest}

The authors declare that they have no conflicts of interest.

\section{Acknowledgments}

The authors would like to appreciate the National Natural Science Foundation of China (Grant numbers: 51775095 and 51605083) and the Fundamental Research Funds for Central Universities (N180703018 and N170308028) for financially supporting the research.

\section{References}

[1] A. Chehouri, R. Younes, A. Ilinca, and J. Perron, "Review of performance optimization techniques applied to wind turbines," Applied Energy, vol. 142, pp. 361-388, 2015.

[2] R. Barrett and A. Ning, "Comparison of airfoil precomputational analysis methods for optimization of wind turbine blades," IEEE Transactions on Sustainable Energy, vol. 7, no. 3, pp. 1081-1088, 2016.
[3] P. J. Schubel and R. J. Crossley, "Wind turbine blade design," Energies, vol. 5, no. 9, pp. 3425-3449, 2012.

[4] B. Li, H. Ma, X. Yu, J. Zeng, X. Guo, and B. Wen, "Nonlinear vibration and dynamic stability analysis of rotor-blade system with nonlinear supports," Archive of Applied Mechanics, vol. 89, no. 7, pp. 1375-1402, 2019.

[5] A. F. P. Ribeiro, A. M. Awruch, and H. M. Gomes, "An airfoil optimization technique for wind turbines," Applied Mathematical Modelling, vol. 36, no. 10, pp. 4898-4907, 2012.

[6] Y. Liu, J. Han, Z. Xue, Y. Zhang, and Q. Yang, "Structural vibrations and acoustic radiation of blade-shafting-shell coupled system," Journal of Sound and Vibration, vol. 463, Article ID 114961, 2019.

[7] S. Yin, D. Yu, Z. Ma, and B. Xia, “A unified model approach for probability response analysis of structure-acoustic system with random and epistemic uncertainties," Mechanical Systems and Signal Processing, vol. 111, pp. 509-528, 2018.

[8] H. S. Toft, L. Svenningsen, W. Moser, J. D. Sørensen, and M. L. Thøgersen, "Assessment of wind turbine structural integrity using response surface methodology," Engineering Structures, vol. 106, pp. 471-483, 2016.

[9] H. Ma, J. Zeng, R. Feng, X. Pang, Q. Wang, and B. Wen, "Review on dynamics of cracked gear systems," Engineering Failure Analysis, vol. 55, pp. 224-245, 2015.

[10] L. Wang, Y. Liu, and Y. Liu, "An inverse method for distributed dynamic load identification of structures with interval uncertainties," Advances in Engineering Software, vol. 131, pp. 77-89, 2019.

[11] Standard, International Electrotechnical Commission, Wind Turbines-Part 1: Design Requirements, Standard, International Electrotechnical Commission, Geneva, Switzerland, 2005.

[12] B. Ernst, H. Schmitt, and J. R. Seume, "Effect of geometric uncertainties on the aerodynamic characteristic of offshore wind turbine blades," Journal of Physics: Conference Series, vol. 555, Article ID 012033, 2014.

[13] J. Zhu and Z. Qiu, "Interval analysis for uncertain aerodynamic loads with uncertain-but-bounded parameters," Journal of Fluids and Structures, vol. 81, pp. 418-436, 2018.

[14] J. D. Sørensen and H. S. Toft, "Probabilistic design of wind turbines," Energies, vol. 3, pp. 241-257, 2010.

[15] S. Wang, Q. Li, and G. J. Savage, "Reliability-based robust design optimization of structures considering uncertainty in design variables," Mathematical Problems in Engineering, vol. 2015, Article ID 280940, 8 pages, 2015.

[16] L. Wang, C. Xiong, J. Hu, X. Wang, and Z. Qiu, “Sequential multidisciplinary design optimization and reliability analysis under interval uncertainty," Aerospace Science and Technology, vol. 80, pp. 508-519, 2018.

[17] P. Wang, S. Wang, X. Zhang et al., "Rational construction of $\mathrm{CoO} / \mathrm{CoF} 2$ coating on burnt-pot inspired 2D CNs as the battery-like electrode for supercapacitors," Journal of Alloys and Compounds, vol. 819, Article ID 153374, 2020.

[18] G. I. Schuëller, H. J. Pradlwarter, and P. S. Koutsourelakis, “A critical appraisal of reliability estimation procedures for high dimensions," Probabilistic Engineering Mechanics, vol. 19, no. 4, pp. 463-474, 2004.

[19] H. Dai and W. Wang, "Application of low-discrepancy sampling method in structural reliability analysis," Structural Safety, vol. 31, no. 1, pp. 55-64, 2009.

[20] H. Zhang, H. Dai, M. Beer, and W. Wang, "Structural reliability analysis on the basis of small samples: an interval quasiMonte Carlo method," Mechanical Systems and Signal Processing, vol. 37, no. 1-2, pp. 137-151, 2013. 
[21] X. Zhang, L. Wang, and J. D. Sørensen, "A novel activelearning function toward adaptive Kriging surrogate models for structural reliability analysis," Reliability Engineering \& System Safety, vol. 185, pp. 440-454, 2019.

[22] X. Zhang, L. Wang, and J. D. Sørensen, “AKOIS: an adaptive Kriging oriented importance sampling method for structural system reliability analysis," Structural Safety, vol. 82, Article ID 101876, 2020.

[23] N. Wiener, "The homogeneous chaos," American Journal of Mathematics, vol. 60, no. 4, pp. 897-936, 1938.

[24] R. Li and R. Ghanem, "Adaptive polynomial chaos expansions applied to statistics of extremes in nonlinear random vibration," Probabilistic Engineering Mechanics, vol. 13, no. 2, pp. 125-136, 1998.

[25] Y. Liu, Q. Meng, X. Yan, S. Zhao, and J. Han, "Research on the solution method for thermal contact conductance between circular-arc contact surfaces based on fractal theory," International Journal of Heat and Mass Transfer, vol. 145, Article ID 118740, 2019.

[26] D. Xiu and G. E. Karniadakis, "The wiener-askey polynomial chaos for stochastic differential equations," SIAM Journal on Scientific Computing, vol. 24, no. 2, pp. 619-644, 2002.

[27] D. Xiu and G. Em Karniadakis, "Modeling uncertainty in steady state diffusion problems via generalized polynomial chaos," Computer Methods in Applied Mechanics and Engineering, vol. 191, no. 43, pp. 4927-4948, 2002.

[28] S. Yin, D. Yu, Z. Luo, and B. Xia, "Unified polynomial expansion for interval and random response analysis of uncertain structure-acoustic system with arbitrary probability distribution," Computer Methods in Applied Mechanics and Engineering, vol. 336, pp. 260-285, 2018.

[29] H. Lu, G. Shen, and Z. Zhu, "An approach for reliability-based sensitivity analysis based on saddlepoint approximation," Proceedings of the Institution of Mechanical Engineers, Part O: Journal of Risk and Reliability, vol. 231, no. 1, pp. 3-10, 2017.

[30] H. Lu, Z. Zhu, and Y. Zhang, "A hybrid approach for reliability-based robust design optimization of structural systems with dependent failure modes," Engineering Optimization, vol. 52, no. 3, pp. 384-404, 2020.

[31] Q. Zhao, X. Chen, Z. D. Ma, and Y. Lin, "Reliability-based topology optimization using stochastic response surface method with sparse grid design," Mathematical Problems in Engineering, vol. 2015, Article ID 487686, 13 pages, 2015.

[32] A. Doostan, R. G. Ghanem, and J. Red-Horse, "Stochastic model reduction for chaos representations," Computer Methods in Applied Mechanics and Engineering, vol. 196, no. 37-40, pp. 3951-3966, 2007.

[33] I. Babuška, F. Nobile, and R. Tempone, "A stochastic collocation method for elliptic partial differential equations with random input data," SIAM Journal on Numerical Analysis, vol. 45, no. 3, pp. 1005-1034, 2007.

[34] L. Wang, C. Xiong, X. Wang, G. Liu, and Q. Shi, "Sequential optimization and fuzzy reliability analysis for multidisciplinary systems," Structural and Multidisciplinary Optimization, vol. 60, no. 3, pp. 1079-1095, 2019.

[35] D. Poljak, S. Šesnić, M. Cvetković et al., "Stochastic collocation applications in computational electromagnetics," Mathematical Problems in Engineering, vol. 2018, Article ID 1917439, 13 pages, 2018.

[36] G. Blatman and B. Sudret, "An adaptive algorithm to build up sparse polynomial chaos expansions for stochastic finite element analysis," Probabilistic Engineering Mechanics, vol. 25, no. 2, pp. 183-197, 2010.
[37] A. Desai and S. Sarkar, "Analysis of a nonlinear aeroelastic system with parametric uncertainties using polynomial chaos expansion," Mathematical Problems in Engineering, vol. 2010, Article ID 379472, 21 pages, 2010.

[38] X. Wei, H. Chang, B. Feng, and Z. Liu, "Sensitivity analysis based on polynomial chaos expansions and its application in ship uncertainty-based design optimization," Mathematical Problems in Engineering, vol. 2019, Article ID 7498526, 19 pages, 2019.

[39] B. Sudret, Uncertainty propagation and sensitivity analysis in mechanical models - Contributions to structural reliability and stochastic spectral methods, Habilitation à diriger des recherches, Université Blaise Pascal, Clermont-Ferrand France, 2007.

[40] T. Zhang, "Sparse recovery with orthogonal matching pursuit under RIP," IEEE Transactions on Information Theory, vol. 57, no. 9, pp. 6215-6221, 2011.

[41] G. Blatman and B. Sudret, "Adaptive sparse polynomial chaos expansion based on least angle regression," Journal of Computational Physics, vol. 230, no. 6, pp. 2345-2367, 2011.

[42] T. Ishigami and T. Homma, "An importance quantification technique in uncertainty analysis for computer models," in Proceedings of the IEEE First International Symposium on Uncertainty Modeling and Analysis, pp. 398-403, Los Alamitos, CA, USA, 1990.

[43] R. Ghanem and P. Spanos, Stochastic Finite Elements: A Spectral Approach, Springer-Verlag, New York, NY, USA, 1991.

[44] X. Zhang, Q. Liu, and H. Huang, "Numerical simulation of random fields with a high-order polynomial based RitzGalerkin approach," Probabilistic Engineering Mechanics, vol. 55, pp. 17-27, 2019.

[45] P. Fuglsang, I. Antoniou, N. Sørensen, and H. Aagaard Madsen, Validation of a Wind Tunnel Testing Facility for Blade Surface Pressure Measurements, International Energy Agency, Paris, France, 1998.

[46] M. Drela, XFOIL: An Analysis and Design System for Low Reynolds Number Airfoils. Low Reynolds Number Aerodynamics, T. J. Mueller, Ed., Springer Berlin Heidelberg, Berlin, Germany, 1989. 